\title{
Article
}

\section{Design and experiments of voltage sensor based on electric field coupling principle and differential input structure}

\author{
Tonghao Zhou $\left.{ }^{1} \mathbb{(}\right)$, Zhe Zhuang ${ }^{1} \mathbb{D}$, Xiu Zhou ${ }^{2}$, Xutao $\mathrm{Wu}^{2}$, Xiuguang $\mathrm{Li}^{2}$, Qing Xiong ${ }^{1, *,+}{ }^{-}$and \\ Shengchang $\mathrm{Ji}^{1, *,+(\mathbb{C})}$ \\ 1 State Key Laboratory of Electrical Insulation and Power Equipment, Xi'an Jiaotong University, Xi'an 710049, \\ China; jsc@mail.xjtu.edu.cn \\ 2 Electric Power Research Institute of State Grid Ningxia Electric Power Co., Ltd., Ningxia 750011, China; \\ zhouxiu007@163.com \\ * Correspondence: xq_xjtu@163.com (Q.X.); jsc@mail.xjtu.edu.cn (S.J.) \\ + These authors contributed equally to this work.
}

\begin{abstract}
Traditional potential transformers have problems of large volume, difficulty in insulation, iron core saturation, ferroresonance overvoltage and poor transient response characteristics. The voltage sensor based on the principle of electric field coupling and differential input structure does not need to contact the measured object or ground, and can avoid the above problems. However, it requires a sufficiently high capacitance between the differential electrodes to obtain sufficient accuracy and a high voltage division ratio. The existing method of using mutual capacitance between the differential electrodes will cause many problems and fail to meet the practical needs. To solve the above problems, this paper innovatively uses multi-layer ceramic capacitor to replace the mutual capacitance and designs a new type of voltage sensor. In addition, by using single bypass small resistance grounding method to increase the input impedance of the differential signal processing circuit, error of the sensor is further reduced. The experimental results show that the sensor has excellent accuracy and great transient response characteristics. The ratio error under power frequency is within $\pm 0.5 \%$ and the phase error is within $1^{\circ}$. The ratio error in the range of $500 \mathrm{~Hz} \sim 30 \mathrm{kHz}$ is within $\pm 5 \%$ and the phase error is within $5^{\circ}$. Moreover, it has the advantages of low cost, miniaturization, flexible shape and easy to adjust the voltage division ratio. These characteristics indicate that the sensor has good voltage measurement and sensor network potential.
\end{abstract}

Keywords: electric field coupling; differential input structure; non-contact voltage measurement; multi-layer ceramic capacitor; transient response

\section{Introduction}

As an important part of power system, voltage measurement plays a decisive role in energy measurement[1-3], relay protection[4-6], overvoltage online monitoring[7,8], and automatic equipment control[9,10]. Its accuracy, real-time performance, and reliability are the basis for ensuring the safe operation of the power system. However, the existing voltage measurement equipments in power system are still dominated by voltage transformers. Among them, the most commonly used are inductive voltage transformer (IVT) and capacitive voltage transformer (CVT). IVT has the problems of large volume, difficulty in insulation and price increase with the increase of voltage level[11], and it is easy to produce ferroresonance overvoltage[12-14] which may cause equipment damage or even power grid outage[15]. Although CVT has better insulation stability, cheaper price, and it is not easy to produce ferroresonance overvoltage[16], since it has internal energy storage components such as capacitors and compensation reactors, these energy needs to be released through the RLC circuit composed of devices and loads. Therefore, the transient characteristics of CVT are poor, and its output voltage can not accurately reflect the change of the primary side voltage[17,18]. The non-contact 
voltage measurement method realized by the principle of electric field coupling does not require direct contact with the measured object and has the advantages of good insulation, simple structure, no overvoltage, wide bandwidth and fast response, etc[19-21]. Because of its good development prospect, it has become a hot spot in current researches.

There are mainly two types of sensors which measure voltage through the principle of electric field coupling: single-ended grounding structure[22,23] and differential input structure[24,25]. The single-ended grounding structure is equivalent to a traditional capacitive voltage divider. It only has one electrode. The high voltage arm is the coupling capacitance between the electrode and the measured object. The low voltage arm is the capacitance between the electrode and the ground, and its output voltage is the potential of the electrode to the ground[26]. This kind of sensor has been widely used in the measurement of very fast transient overvoltage (VFTO) in gas insulated switchgear (GIS)[21,27], but it needs to be coupled to the measured object as well as be grounded, which greatly limits its application scope. To solve this problem, some scholars have proposed the differential input structure which uses the coupling capacitances of two electrodes to the ground and the measured object as the high voltage arm, and the mutual capacitance between the two electrodes as the low voltage arm, so the sensor is not need to be grounded[28-30]. However, the sensor with differential input structure requires a large capacitance between the two differential electrodes to meet the self-integration condition and have a high voltage division ratio[31]. In the existing researches, methods such as expanding the equivalent area of the electrodes and filling dielectric between the electrodes are generally used to increase the mutual capacitance between the electrodes[25,31,32]. However, the capacitance obtained by these ways is still small and inaccurate. Besides, these methods will cause problems such as the sensor becomes larger, the mutual capacitance is easily to be interfered by temperature and humidity, the voltage division ratio is difficult to adjust and the shape of sensor is relatively fixed, etc.

In this paper, a new type of voltage sensor is designed using multi-layer ceramic capacitor to replace the mutual capacitance between the two electrodes and using the method of single bypass small resistance grounding to increase the input impedance of differential signal processing circuit. The sensor has the advantages of miniaturization, easy to adjust the voltage division ratio, flexible shape and low cost, which could successfully solve many problems in existing similar sensors. Moreover, steady-state and transient experiments are carried out to investigate the frequency response characteristics and accuracy of the sensor.

\section{Principle and Design of Sensor}

\subsection{The Principle of Electric Field Coupling and Differential Input Structure}

The voltage sensor, based on electric field coupling principle and differential input structure, is mainly composed of metal electrode 1 , electrode 2 and differential signal processing circuit. The schematic diagram is shown in Figure 1. $C_{m 1}$ and $C_{m 2}$ are the coupling capacitances of electrode 1 and electrode 2 to the measured object respectively. $C_{s 1}$ and $C_{s 2}$ are the coupling capacitances of electrode1 and electrode 2 to the ground respectively. $C_{m 0}$ is the capacitance between electrode 1 and electrode 2. $R_{n}$ is the input impedance of the differential signal processing circuit. The transfer function of the sensor can be expressed as:

$$
H(s)=\frac{V(s)}{\varphi(s)}=\frac{s C_{1} R_{n}}{s C_{2} R_{n}+1}
$$

Where, $V(s)$ is the potential difference between the two electrodes; $\varphi(s)$ is the potential difference between the measured object and the ground; $C_{1}=\frac{C_{m 1} C_{s 2}-C_{m 2} C_{s 1}}{C_{m 1}+C_{m 2}+C_{s 1}+C_{s 2}} ; C_{2}=\frac{1}{C_{m 1}+C_{s 1}}+\frac{1}{C_{m 2}+C_{s 2}}+C_{m 0}$.

Therefore, the amplitude frequency response function of the sensor is: 


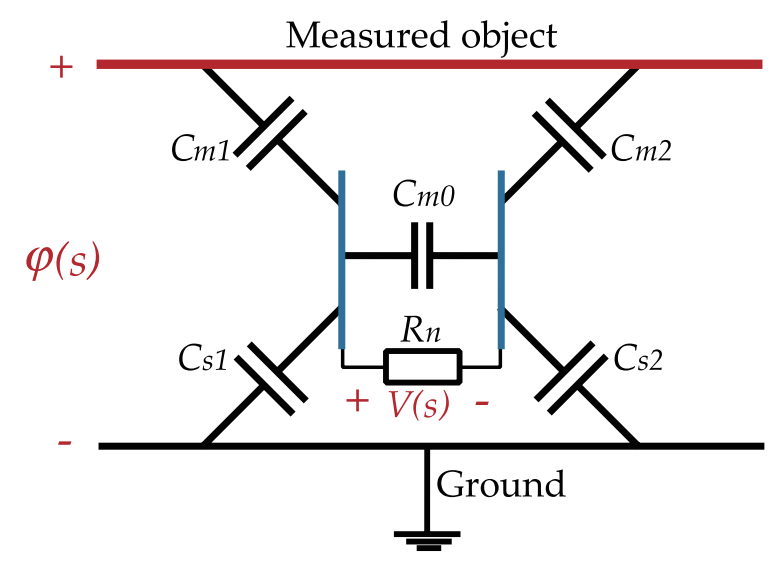

Figure 1. Schematic diagram of the sensor.

$$
|H(\omega)|=\frac{C_{1} R_{n}}{\sqrt{\left(C_{2} R_{n}\right)^{2}+\frac{1}{\omega^{2}}}}
$$

Where, $\omega$ is the equivalent angular frequency of the measured voltage, and $\omega=2 \pi f, f$ is the frequency of the measured voltage.

The phase frequency response function of the sensor is:

$$
\angle H(\omega)=\arctan \frac{1}{C_{2} R_{n} \omega}
$$

The condition for the sensor in self-integration mode is:

$$
C_{2} R_{n} \gg \frac{1}{\omega}
$$

When self-integration condition is satisfied, it can be considered that its voltage division ratio is $\frac{C_{1}}{C_{2}}$, phase difference $|\angle H(\omega)|=0$. Therefore, improving the values of $C_{2}$ and $R_{n}$ is the key to make the sensor meet the self-integration condition and reduce error. Since the values of $C_{m 1}, C_{m 2}, C_{s 1}, C_{s 2}$ are picofarad level, they can be ignored relative to the value of $C_{m 0}$ (as will be explained later, its value is generally nanofarad level), according to the definition of $C_{2}$ in formula (1), it can be considered that:

$$
C_{2} \approx C_{m 0}
$$

Meanwhile, since the input impedance of the differential signal processing circuit will affect the bias current of the operational amplifier and thus affect the frequency response of the circuit, it has certain limitations to increase $R_{n}$. Therefore, increasing $C_{m 0}$ is the main method to make the sensor satisfy the self-integration condition and increase the voltage division ratio.

In the existing researches, $C_{m 0}$ is the mutual capacitance between the two electrodes, its value is generally several nanofarads which means the voltage division ratio of the sensor is several thousand to one[29], which can't meet the measurement requirements of ultra-high voltage equipments. To solve this problem, high performance intergrated capacitor is used to replace the traditional mutual capacitance between the electrodes, and the value of $C_{m 0}$ can be changed conveniently and accurately. The multi-layer ceramic capacitor used in this sensor has advantages of small volume, high reliability, relatively inexpensive[33] and low loss. Its operating temperature range is within $-55^{\circ} \mathrm{C} \sim 125^{\circ} \mathrm{C}$, and the temperature coefficient is only $\pm 30 \mathrm{ppm} /{ }^{\circ} \mathrm{C}$ in this range. The influnce of ambient temperature on the capacitance can be almostly ignored. 


\subsection{Differential Signal Processing Circuit}

Since the sensor adopts differential input structure, as shown in Figure 1, the potentials of electrode 1 and electrode 2 are both unknown floating potentials. It is necessary to use the differential signal processing circuit to convert the potential difference between the two electrodes into single-ended signal relative to the circuit reference ground potential. The differential signal processing circuit used in this sensor is shown in Figure 2.

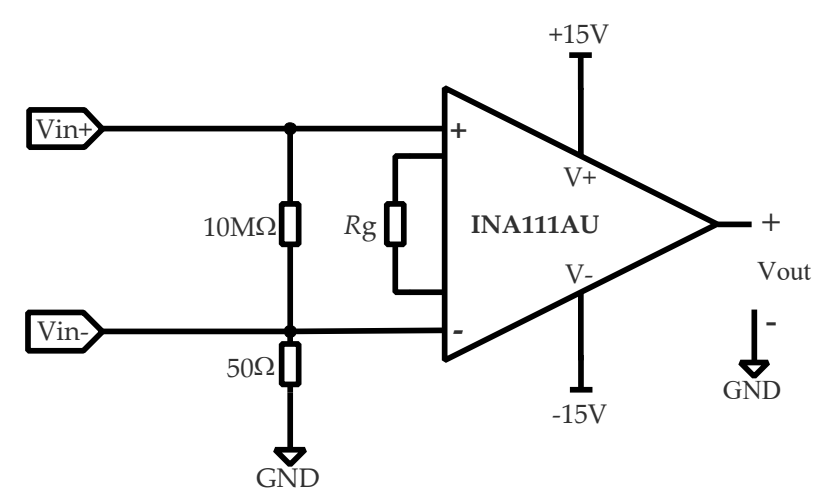

Figure 2. Structure diagram of differential signal processing circuit.

The chip used in the circuit is INA111AU instrumentation amplifier[34] produced by Texas Instruments and powered by $\pm 15 \mathrm{~V}$. To ensure that the amplifier has a DC operating point and works in the linear operating region, the two input ends of the amplifier should have DC paths to the reference ground of the printed circuit board (PCB). Considering that the max bias current of INA111AU is only $\pm 20 \mathrm{pA}$, large resistance can be appropriately selected to provide DC paths and input impedance. However, if the resistance is too large, the voltage generated by the bias current flowing through the resistors will be regarded as part of the input voltage, thus the output voltage has a large DC bias.

In this circuit, a $10 \mathrm{M} \Omega$ resistor is used between the two input ends to provide the input impedance, and a $50 \Omega$ resistor is grounded to provide the DC paths. The minimum input common-mode voltage range of the chip is only $\pm 10 \mathrm{~V}$. When using large grounding resistance, the common-mode voltage between the PCB and the electrodes will be too high, which casues the chip not working properly. The single bypass small resistance grounding method can effectively solve this problem and make the circuit obtain higher input impedance. Therefore, the single bypass small resistance grounding is adopted instead of traditional double bypasses large resistance grounding.

\subsection{Parameter Selection and Fabrication of the Sensor}

Since the method proposed in this paper uses multi-layer ceramic capacitor to replace the mutual capacitance between the electrodes, there are no special requirements for the size and shape of the two electrodes. However, if the size of two electrodes are equal, the values of $C_{m 1}$ and $C_{m 2}, C_{s} 1$ and Cs2 may be similar or even equal, and according to formula (1), the potential difference between the two electrodes is almost zero, so the size of electrode 1 and electrode 2 should be designed to have sufficient difference. The physical maps of electrodes designed in this paper are shown in Figure 3. The two electrodes are respectively located on the front side and the reverse side of the PCB, the thickness of PCB is $1.2 \mathrm{~mm}$. The size of the front electrode is $30 \mathrm{~mm}$ inner diameter and $80 \mathrm{~mm}$ outer diameter. The size of the reverse electrode is $50 \mathrm{~mm}$ inner diameter and $80 \mathrm{~mm}$ outer diameter. 


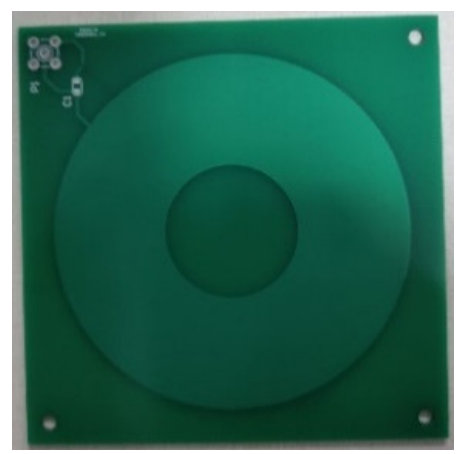

(a)

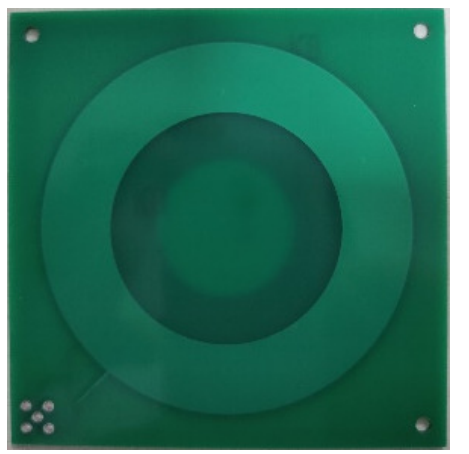

(b)

Figure 3. Physical maps of electrodes: (a) Front side; (b) Reverse side.

To select the value of the multi-ceramic capacitor on the sensor, the calculation result of formula (3) can be used as a reference. In this paper, $R_{n}=10 \mathrm{M} \Omega$. If $C_{m 0}$ is $4.7 \mathrm{nF}$, the calculation result of phase error is $3.87^{\circ}$ at $50 \mathrm{~Hz}$, and if $C_{m 0}$ is $47 \mathrm{nF}$, the result is $0.388^{\circ}$, which can basically meet the general measurement requirements. Therefore, without special instructions, the sensor uses a $47 \mathrm{nF}$ capacitor as $C_{m 0}$.

When using a capacitor of $47 \mathrm{nF}$ as $C_{m 0}$, the measured voltage division ratio is hundreds of thousands to one. In the laboratory, the voltage of measured objects is generally several kilovolts or even lower, and the output voltage of the sensor is only tens of millivolts. Due to the noise of differential circuit, oscilloscope and other electronic components, too small voltage will lead to the decrease of signal-to-noise ratio, so it is necessary to provide gain in the differential circuit. In Figure 2, $R g$ is the gain adjusting resistor, the relationship between the gain $(G)$ of instrumentation amplifier and $R g$ is:

$$
\mathrm{G}=1+\frac{50 \mathrm{k} \Omega}{\operatorname{Rg}}
$$

According to the datasheet of the amplifier[34], high gain has negative effect on its frequency response, and ten times of gain has already meet the requirement of the experiments in this paper. Therefore, a resistor of $5.6 \mathrm{k} \Omega$ is used as $\mathrm{Rg}$ and the theoretical gain is 9.93 . Figure 4 shows the frequency response of the differential circuit, and in the range of $20 \mathrm{~Hz} \sim 100 \mathrm{kHz}$. The differential circuit has a stable response.

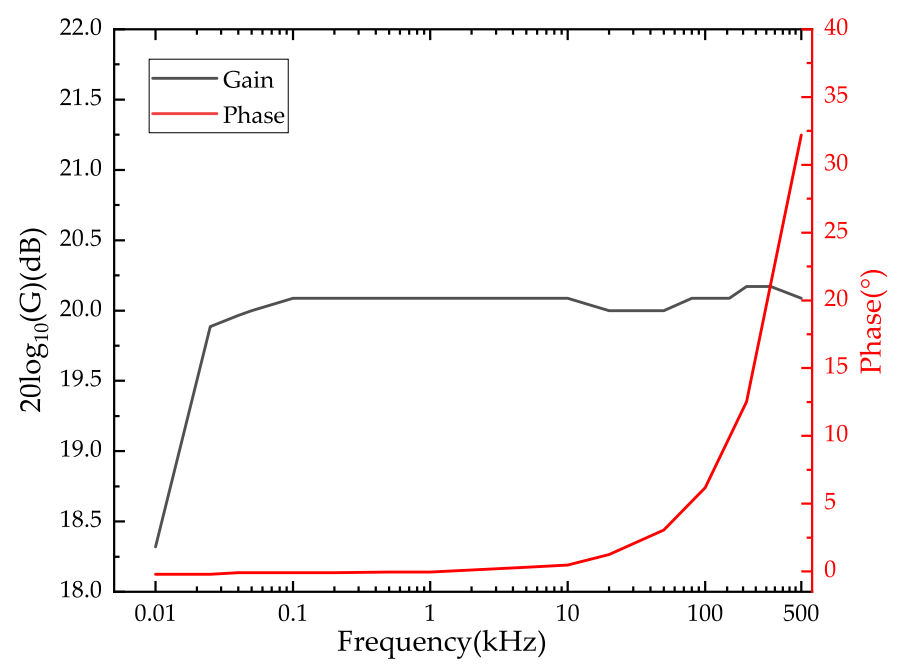

Figure 4. Frequency response of the differential circuit. 


\section{Experiments and Results Analysis}

\subsection{Power Frequency Steady-State Experiments}

The platform of power frequency experiments is shown in Figure 5. The transformer is used to generate $50 \mathrm{~Hz}$ power frequency voltage, and the capacitive voltage divider with a voltage division ratio of 1000:1 is used to provide the amplitude and phase reference. The voltage sensor is placed on the post insulator which is tens of centimeters to several meters away from the high voltage terminal of the transformer. Both the voltage sensor and the capacitive voltage divider are connected to the Tek DPO4014B oscilloscope.

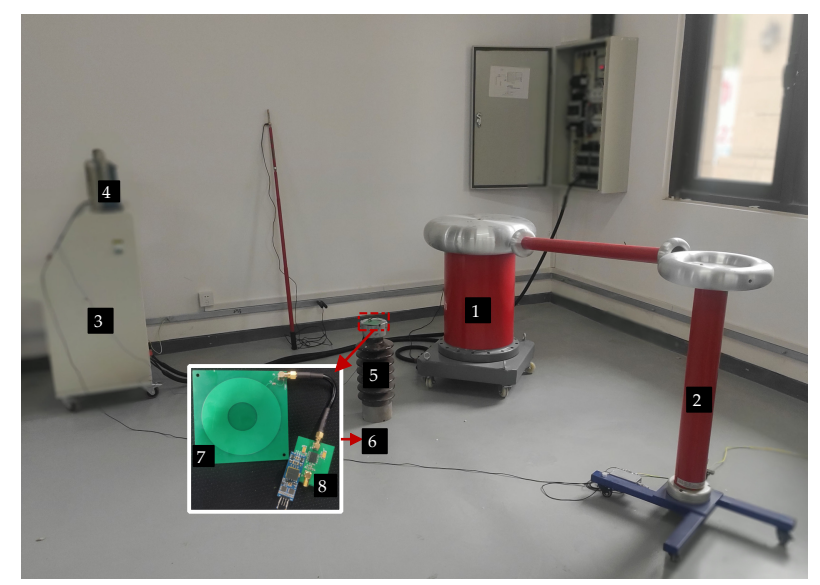

1. Transformer 2. Capacitive voltage divider 3. Voltage regulator

4. Oscilloscope 5. Post insulator 6. Volatge sensor

7. Differential electrodes 8. Differential signal processing circuit

(a)

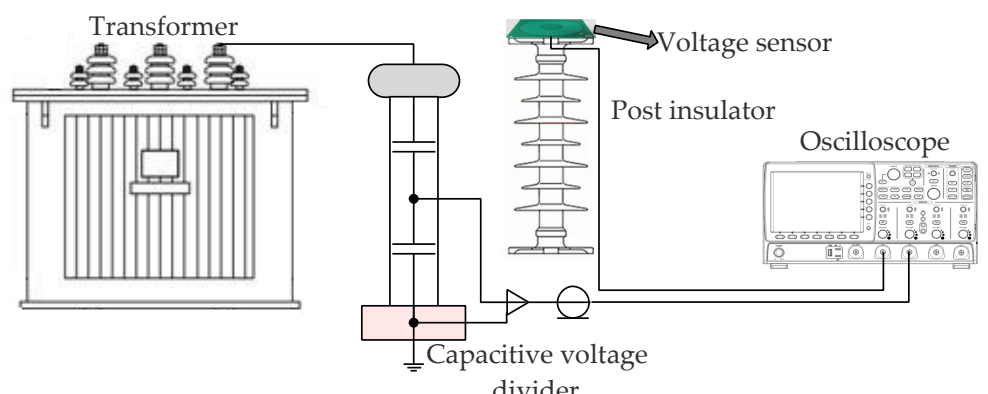

(b)

Figure 5. Power frequency experimental platform: (a) Physical map; (b) Schematic diagram.

To obtain the voltage division ratio and linearity of the voltage sensor, under the case that the distance between the sensor and the high voltage terminal is $153 \mathrm{~cm}$, the transformer works in no-load state, the output voltage of the transformer is adjusted through the voltage regulator, and the output voltage of capacitive voltage divider and voltage sensor are recorded at the same time. Figure 6 shows the measurement points of this experiment and the fitted first-order curve.

The expression of the fitted curve is $y=0.0237 x-0.0007$. The correlation coefficient of the transformer and the sensor output voltage is 1 . The result shows the voltage sensor has excellent linearity. According to the fitted curve, the rated voltage division ratio of the sensor is 42194.09, and then the ratio error of the sensor is calculated and shown in Table 1 . The definition of the ratio error $(\varepsilon \%)$ is:

$$
\varepsilon \%=\frac{K_{n} U_{s}-U_{p}}{U_{p}} \times 100 \%
$$


Where, $K_{n}$ is the rated voltage division ratio of the sensor; $U_{p}$ is the output voltage of the transformer; $U_{s}$ is the output voltage of the voltage sensor.

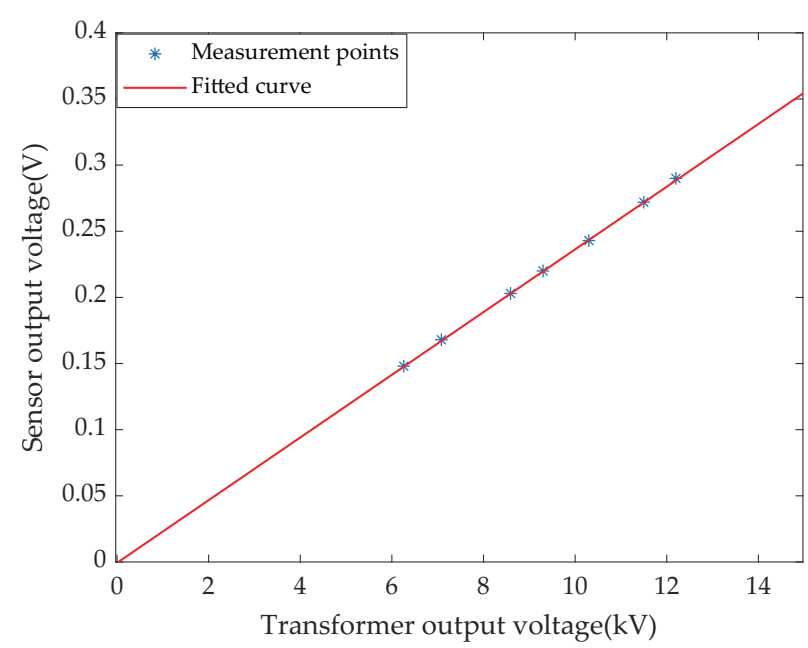

Figure 6. Measurement points and fitted curve.

Table 1. Calculation result of ratio error.

\begin{tabular}{ccr}
\hline$U_{p}(\mathbf{k V})$ & $U_{s}(\mathrm{~V})$ & \multicolumn{1}{c}{$\varepsilon \%$} \\
\hline 6.26 & 0.148 & -0.244 \\
7.08 & 0.168 & 0.122 \\
8.59 & 0.203 & -0.286 \\
9.30 & 0.220 & -0.186 \\
10.3 & 0.243 & -0.455 \\
11.5 & 0.272 & -0.202 \\
12.2 & 0.290 & 0.297 \\
\hline
\end{tabular}

The experimental result shows that the voltage sensor has high voltage division ratio and excellent accuracy, with the ratio error less than $\pm 0.5 \%$.

For phase error, the function of oscilloscope is used to measure the phase difference between two channels, as shown in Figure 7. Channel 1 is the output voltage of the sensor. Channel 2 is the output

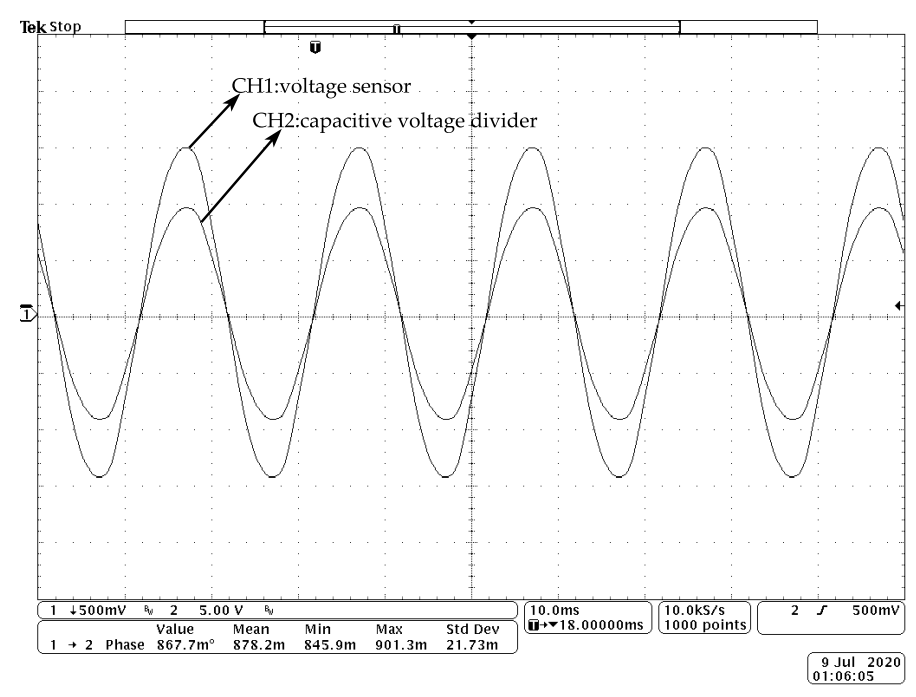

Figure 7. Power frequency phase error of the sensor. 
voltage of capacitive voltage divider and is used as the reference waveform of the transformer output voltage.

In addition, to compare the measured phase error with the theoretical phase error and understand the effect of $C_{m 0}$ value on the phase error, the experiments are also carried out in the cases of $C_{m 0}=4.7 \mathrm{nF}$, $100 \mathrm{nF}$ and $220 \mathrm{nF}$ respectively. The experimental results and the comparison between theory and experiment are shown in Table 2. It can be seen that the phase error of measuring is close to but slightly larger than the theoretical error. This is because that the sensor can also couple to the electric field generated by the other nearly divices, stray parameters of the components and circuits. Moreover, the capacitive voltage divider also has certain error.

Table 2. Results of phase error.

\begin{tabular}{cccc}
\hline$C_{m 0}(\mathrm{nF})$ & Theoretical phase error & Measured phase error & Extra error \\
\hline 4.7 & $3^{\circ} 52^{\prime}$ & $4^{\circ} 15^{\prime}$ & $23^{\prime}$ \\
47 & $23^{\prime}$ & $42^{\prime}$ & $19^{\prime}$ \\
100 & $11^{\prime}$ & $28^{\prime}$ & $17^{\prime}$ \\
220 & $5^{\prime}$ & $8^{\prime}$ & $3^{\prime}$ \\
\hline
\end{tabular}

Since the values of coupling capacitances $C_{m 1}, C_{m 2}, C_{s 1}, C_{s 2}$ are all related to the distance between the sensor and the measured object, it is necessary to investigate the relationship between the distance and the voltage division ratio of the sensor. For this part of experiment, first, the height of both the post insulator and the transformer high voltage terminal are measured, and then change the distance between the post insulator and the transformer, the distance and the voltage division ratio are recorded. Finally, the voltage division ratios under different distance between the sensor and the high voltage terminal are calculated. The result is shown in Figure 8. It can be seen that the distance between the sensor and the measured object has a significant impact on the voltage division ratio. If need to obtain a fixed voltage division ratio, the position of the sensor should be fixed.

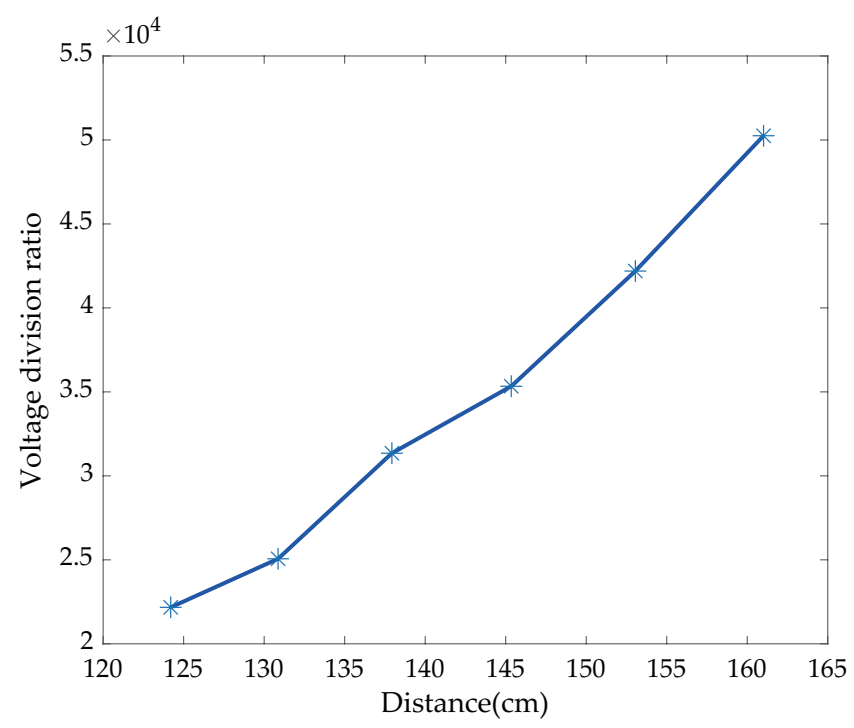

Figure 8. Relationship between sensor voltage division ratio and distance.

According to the results of the power frequency steady-state experiments, it can be seen that the voltage sensor designed in this paper has excellent linearity and accuracy. The ratio difference is within $0.5 \%$. Increasing the value of $C_{m 0}$ can increase the voltage division ratio and reduce the phase error. When $C_{m 0}$ is equal or larger than $47 \mathrm{nF}$, the phase error can be less than $1^{\circ}$. However, due to the principal of electric field coupling, the voltage division ratio is very sensitive to the position. If need 
to accurately measure the voltage amplitude, the sensor should be calibrated and placed at a fixed position.

\subsection{High Frequency Steady-state Experiments}

To study the amplitude frequency and phase frequency characteristics of the sensor, the high frequency steady-state experimental platform is built as shown in Figure 9. The high-voltage power amplifier is Trek Model 30/20A, which has a fixed gain of $3000 \mathrm{~V} / \mathrm{V}$, and the small signal bandwidth can reach $30 \mathrm{kHz}$. The signal generator is RIGOL DG4102 with a bandwidth of $100 \mathrm{MHz}$, and the model of high-voltage probe is Tek P6015A with a voltage division ratio of $1000: 1$ and a bandwidth of $75 \mathrm{MHz}$. The output of amplifier is connected to a load capacitor, the grading ring of the capacitor is used as a high-voltage terminal, and the voltage sensor is placed $25 \mathrm{~cm}$ away from the grading ring. The high-voltage probe is used to provide the phase and amplitude reference of the amplifier output voltage.

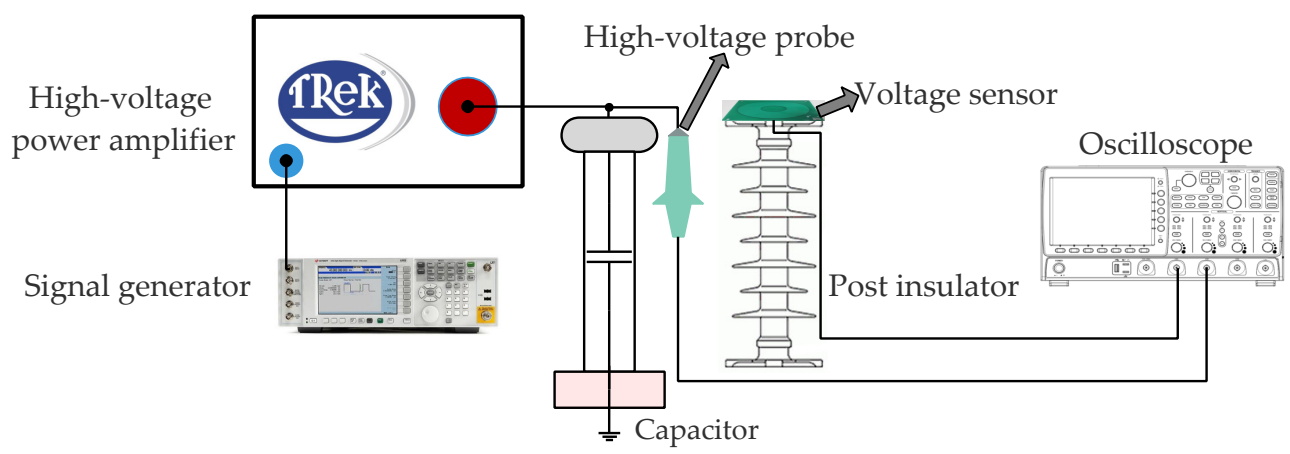

Figure 9. Schematic diagram of high frequency experimental platform

In this experiment, the signal generator outputs a sinusoidal signal of $0.1 \mathrm{~V}$, and the output voltage of the amplifier is only about $300 \mathrm{~V}$. Therefore, to obtain higher sensitivity, the sensor uses a $4.7 \mathrm{nF}$ capacitor as $C_{m 0}$. The measurement results at different frequencies are shown in Table 3 . The average voltage division ratio of the sensor is 3569.01 , which is regarded as the rated voltage division ratio to calculate the ratio error.

Table 3. Results of high frequency response.

\begin{tabular}{cccccc}
\hline $\begin{array}{c}\text { Frequency } \\
(\mathbf{k H z})\end{array}$ & $\begin{array}{c}\text { Output voltage } \\
\text { of the sensor } \\
(\mathbf{m V})\end{array}$ & $\begin{array}{c}\text { Output voltage } \\
\text { of the probe } \\
\mathbf{( m V )}\end{array}$ & Phase error & $\begin{array}{c}\text { Voltage division } \\
\text { ratio }\end{array}$ & Ratio error\% \\
\hline 0.5 & 86.1 & 302.8 & $49^{\prime}$ & 3516.84 & -1.46 \\
1 & 87.8 & 302.7 & $25^{\prime}$ & 3448.00 & -3.39 \\
5 & 95.4 & 326.2 & $-2^{\circ} 6^{\prime}$ & 3419.29 & -4.20 \\
10 & 105.8 & 376.5 & $-2^{\circ} 54^{\prime}$ & 3557.59 & -0.32 \\
20 & 113.5 & 423.2 & $-3^{\circ} 54^{\prime}$ & 3728.63 & 4.47 \\
30 & 70.8 & 265.0 & $-4^{\circ} 42^{\prime}$ & 3744.00 & 4.90 \\
\hline
\end{tabular}

It can been seen that the sensor has a great high frequency response in the range of $500 \mathrm{~Hz} \sim 30 \mathrm{kHz}$. The phase error is within $5^{\circ}$, and the ratio error is within $\pm 5 \%$. For the error sources, in addition to the reasons analyzed in 3.1, it can be seen from Figure 4 that at high frequency, the error of the differential signal processing circuit itself, especially the phase error, can't be ignored. Besides, with the increase of frequency, the dielectric constant will decrease, leading to the value of capacitor decreases, which is one reason for the increase of the voltage division ratio under high frequency.

Therefore, the accuracy of the sensor can be improved by using components with better frequency response characteristics, using multiple capacitors paralleled to form a squirrel cage structure and using twisted pair connection between the electrodes and the differential circuit to reduce inductance. 


\subsection{Transient Characteristics Experiments}

Power system has strict requirements on the transient characteristics of the voltage sensor. Whether the transient signal waveform can be quickly and accurately responsed without oscillation is one of the important performance indicators of the sensor. In view of the sensor has good high frequency response characteristics, the transient response characteristics of the sensor are tested through transformer switch operation and cable arc discharge.

The power frequency experimental platform shown in Figure 5 is also used to carry out the transformer switch operation experiment. The closing operation is carried out after preloading $6.5 \mathrm{kV}$ voltage on the voltage regulator, and the transient response waveforms of the voltage sensor and the capacitive voltage divider are shown in Figure 10(a). After the transformer operates stably for a period of time, making the voltage regulator disconnect with the transformer, and the transient response waveforms are shown in Figure 10(b). According to the measurement results, for the switch operation, the response waveform of the voltage sensor is consistent with the capacitive voltage divider, and no oscillation occurs.

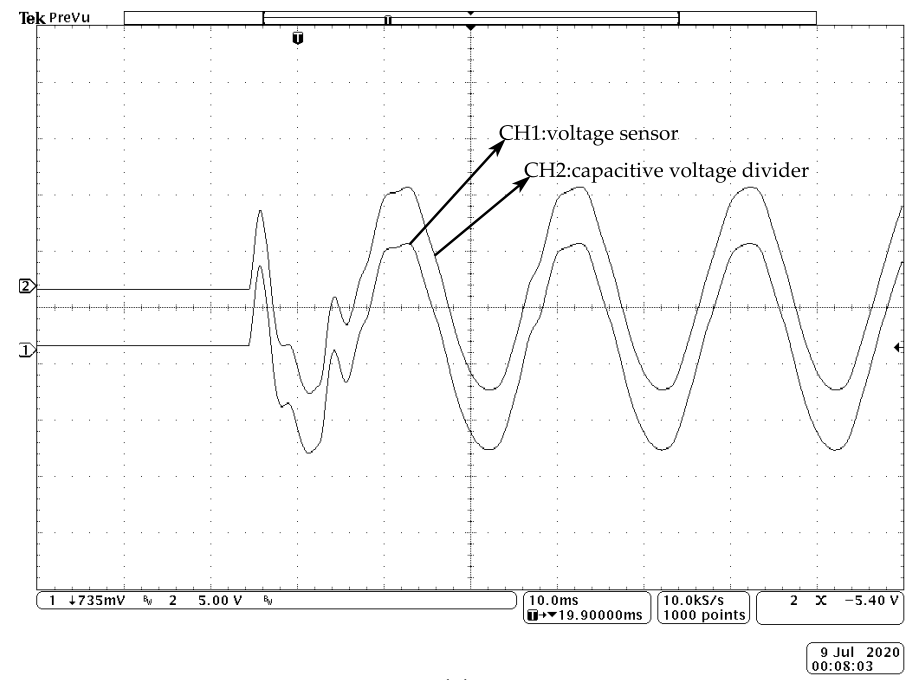

(a)

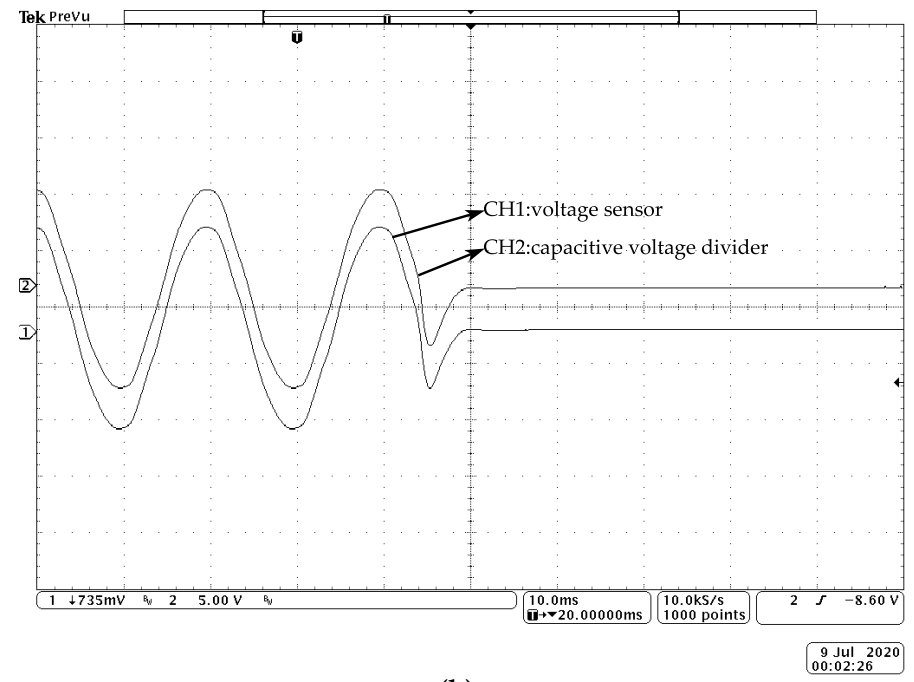

(b)

Figure 10. Waveforms of transformer switch operation: (a) Waveforms of transformer closing operation; (b) Waveforms of transformer opening operation.

The experimental platform shown in Figure 11 is used for the measurement of cable arc discharge. There is an air gap between cable 1 and cable 2, when the output voltage of the transformer is increased 
to a certain value, an arc discharge will occur in the gap. The voltage sensor is placed on the post insulator about $50 \mathrm{~cm}$ away from the air gap.

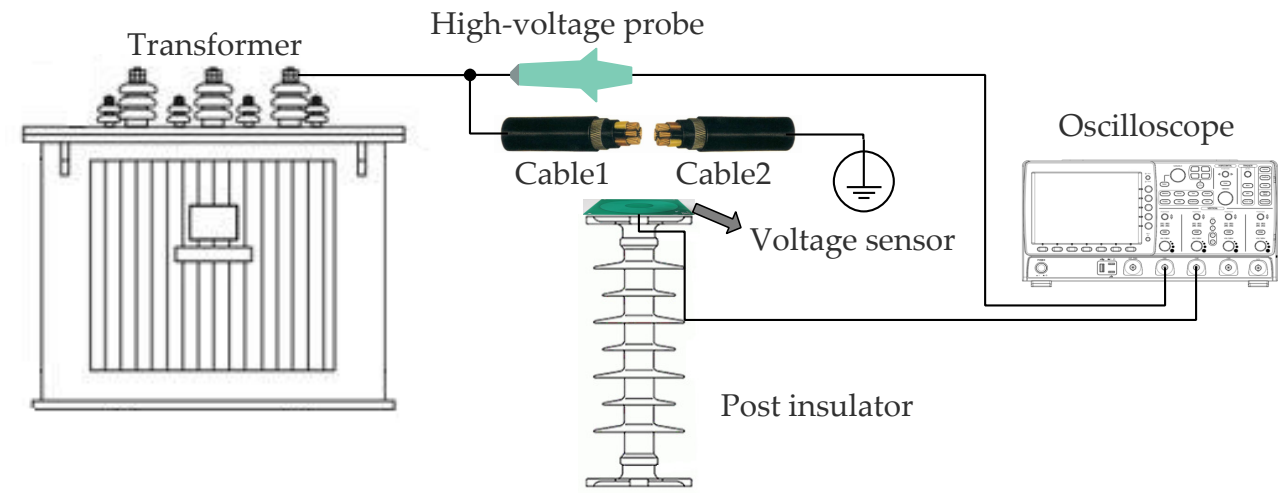

Figure 11. Schematic diagram of cable arc discharge experimental platform.

The cable arc discharge waveforms measured according to this experimental platform are shown in Figure 12. The output voltage waveforms of high-voltage probe and the voltage sensor are basically the same.

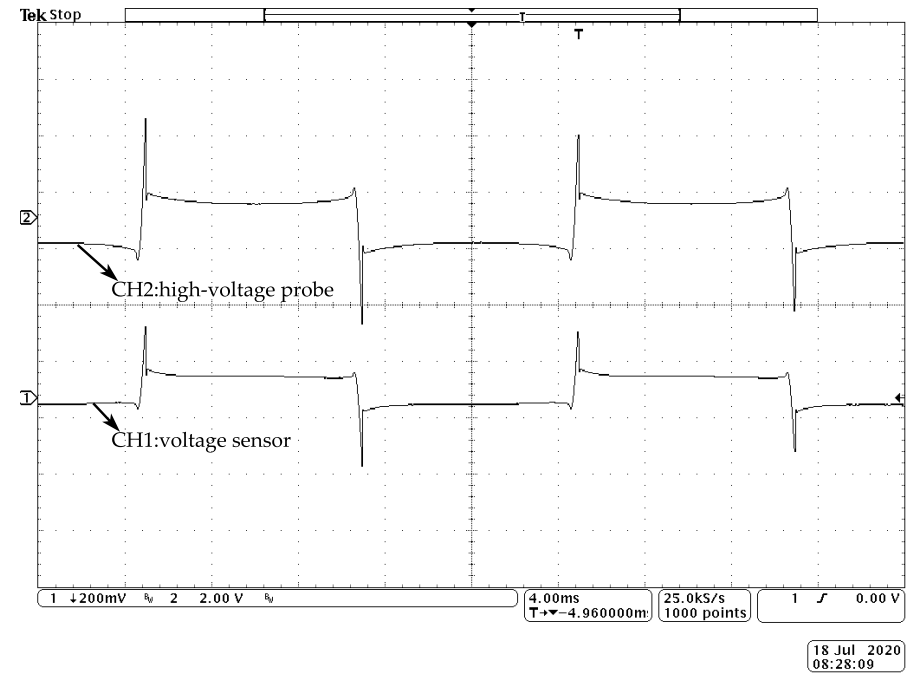

Figure 12. Waveforms of cable arc discharge.

The results of transient characteristics experiments show that the voltage sensor designed in the paper has great transient response characteristics, which can quickly and accurately respond to the transient signal without oscillation.

\section{Discussion}

Based on the experimental results and the advantages of the designed sensor, for the practical application of the sensor, the following situations are given:

1. Used to measure voltage waveform and amplitude: When the sensor is placed in a fixed position near the measured object and calibrated, as long as the environment does not change significantly, it has a fixed voltage division ratio and can accurately measure the waveform and amplitude.

2. Used to improve the transient response characteristics of the existing voltage transformers: When used together with the voltage transformer, the sensor can be calibrated in real time and when overvoltage occurs, the response signal of the sensor can be used to replace the response signal of the voltage transformer. In this case, the sensor does not need a fixed position and is not vulnerable to the external environment change, so it has higher accuracy. 
3. Used to measure the voltage phase: For the measurement of leakage current and partial discharge, the voltage phase of the measured object is one of the important information. The sensor can measure the voltage phase conveniently and accurately without calibration. Therefore, it has broad application prospects in the above aspects.

4. Used for partial discharge measurement: If the parameters of the sensor are changed appropriately, the power frequency voltage can be filtered out and only the high frequency voltage information can be measured. It is a promising research direction for on-line partial discharge monitoring.

5. Used for sensor network: In view of the advantages of small size, low power consumption, flexible shape and low cost, the sensor has application prospects in sensor network.

\section{Conclusions}

This paper proposes a new type of non-contact voltage sensor and describes its structural design in detail. Based on the principal of electric field coupling and the differntial input structure, the sensor innovatively uses multi-layer ceramic capacitor to replace the mutual capacitance between the two differential electrodes, and uses single bypass small resistance grounding method to increase the input impedance of the differential circuit, thus solving many problems of previous similar sensors.

Steady-state and transient characteristics experiments of the sensor are carried out and show that the sensor has excellent accuracy and great transient response characteristics. The ratio error under power frequency is within $\pm 0.5 \%$ and the phase error is within $1^{\circ}$. The ratio error in the range of $500 \mathrm{~Hz} \sim 30 \mathrm{kHz}$ is within $\pm 5 \%$ and the phase error is within $5^{\circ}$. Meanwhile, the experimental results also show that the sensor has the problem of error increase at high frequency. In future research, it is possible to reduce the error and increase its bandwidth by using components with better frequency response characteristics and optimizing the structure of the sensor.

In summary, the sensor designed in this paper has excellent characteristics. These characteristics demonstrate the research and application potential of voltage measurement and sensor network from this sensor.

Author Contributions: Methodology, T.Z. and Z.Z.; validation, X.Z., X.W. and X.L.; writing-original draft preparation, T.Z.; writing-review and editing, Q.X. and S.J.; project administration, S.J.; funding acquisition, Q.X. All authors have read and agreed to the published version of the manuscript.

Funding: This work is partially supported by National Natural Science Foundation of China (No. 52077167).

Acknowledgments: Thanks to Prof. Xia Wang and student Shuai Liu for providing Trek high-voltage power amplifier and related technical support for this research.

Conflicts of Interest: The authors declare no conflict of interest.

\section{References}

1. Martinez, E.V.; De La O Serna, J.A. Smart grids Part 1: Instrumentation challenges. IEEE Instrum. Meas. Mag. 2015, 18, 6-9. doi:10.1109/MIM.2015.7016673.

2. Apse-Apsitis, P.; Avotins, A.; Ribickis, L. Bidirectional DC/AC energy flow measurement. 2015 IEEE 5th International Conference on Power Engineering, Energy and Electrical Drives (POWERENG), 2015, pp. 465-468. doi:10.1109/PowerEng.2015.7266362.

3. Nayusrizal, N.; Untari, I.; Putra, A.A.; Hudaya, C. Effects of Harmonic Distortions on the Accuracy of Energy and Power Losses Measurements in a Water Treatment Industry. 2019 IEEE 2nd International Conference on Power and Energy Applications (ICPEA), 2019, pp. 277-281. doi:10.1109/ICPEA.2019.8818547.

4. Tajdinian, M.; Allahbakhshi, M.; Biswal, S.; Malik, O.P.; Behi, D. Study of the Impact of Switching Transient Overvoltages on Ferroresonance of CCVT in Series and Shunt Compensated Power Systems. IEEE Trans. Industr. Inform. 2020, 16, 5032-5041. doi:10.1109/TII.2019.2951332.

5. Shin, M.C.; Park, C.W.; Kim, J.H. Fuzzy logic-based relaying for large power transformer protection. IEEE Trans. Power Deliv. 2003, 18, 718-724. doi:10.1109/TPWRD.2003.813598. 
6. Ramesh, K.; Sushama, M. Power transformer protection using fuzzy logic based-relaying. 2014 International Conference on Advances in Electrical Engineering (ICAEE), 2014, pp. 1-7. doi:10.1109/ICAEE.2014.6838528.

7. Wang, J.; Yang, Q.; Sima, W.; Yuan, T.; Zahn, M. A Smart Online Over-Voltage Monitoring and Identification System. Energies 2011, 4, 599-615. doi:10.3390/en4040599.

8. Yao, C.; Zhang, X.; Du, L.; Mi, Y.; Sun, C. Online lightning and internal overvoltages monitoring system in $10 \mathrm{kV}$ distribution networks. Eur. Trans. Electr. Power 2011, 21, 282-292. doi:10.1002/etep.439.

9. Quach, N.T.; Chae, S.H.; Song, S.H.; Kim, E.H. Frequency and Voltage Control Strategies of the Jeju Island Power System Based on MMC-HVDC Systems. J. Power Electron. 2018. doi:10.6113/JPE.2018.18.1.204.

10. Yoshida, H.; Kawata, K.; Fukuyama, Y.; Takayama, S.; Nakanishi, Y. A particle swarm optimization for reactive power and voltage control considering voltage security assessment. IEEE Trans. Power Syst. 2000, 15, 1232-1239. doi:10.1109/59.898095.

11. Fang, C.; Li, W.; Wang, J.; Yu, J.; Cheng, C.; Hou, X. 10kV electronic voltage transformer based on resistor divider. Trans. China Electrotech. Soc. 2007, 22, 58-63. doi:10.19595/j.cnki.1000-6753.tces.2007.05.011.

12. Abbasi, A.; Fathi, S.H.; Gharehpatian, G.B.; Gholami, A.; Abbasi, H.R. Voltage transformer ferroresonance analysis using multiple scales method and chaos theory. Complexity 2013, 18, 34-45. doi:10.1002/cplx.21456.

13. Andrei, R.G.; Halley, B.R. Voltage Transformer Ferroresonance from an Energy Transfer Standpoint. IEEE Power Eng. Rev. 1989, 9, 63-63. doi:10.1109/MPER.1989.4310817.

14. Olguín-Becerril, M.A.; Angeles-Camacho, C.; Fuerte-Esquivel, C.R. Ferroresonance in subharmonic 3rd mode in an inductive voltage transformer, a real case analysis. Int. J. Electr. Power Energy Syst. 2014, 61, 318-325. doi:10.1016/j.ijepes.2014.03.057.

15. Radmanesh, H. Effect of Circuit Breaker Shunt Resistance on Chaotic Ferroresonance in Voltage Transformer. Adv. Electr. Comput. Eng. 2010, 10, 71-77. doi:10.4316/AECE.2010.03012.

16. Chen, B.; Du, L.; Liu, K.; Chen, X.; Zhang, F.; Yang, F. Measurement Error Estimation for Capacitive Voltage Transformer by Insulation Parameters. Energies 2017, 10, 357. doi:10.3390/en10030357.

17. Seljeseth, H.; Saethre, E.; Ohnstad, T.; Lien, I. Voltage transformer frequency response. Measuring harmonics in Norwegian $300 \mathrm{kV}$ and $132 \mathrm{kV}$ power systems. 8th International Conference on Harmonics and Quality of Power. Proceedings (Cat. No.98EX227), 1998, Vol. 2, pp. 820-824. doi:10.1109/ICHQP.1998.760148.

18. Xiao, Y.; Fu, J.; Hu, B.; Li, X.; Deng, C. Problems of voltage transducer in harmonic measurement. IEEE Trans. Power Deliv. 2004, 19, 1483-1487. doi:10.1109/TPWRD.2004.829947.

19. Yarovoy, A.; de Jongh, R.; Ligthart, L. Ultra-wideband sensor for electromagnetic field measurements in time domain. Electron. Lett. 2000, 36, 1679-1680. doi:10.1049/el:20001171.

20. Wagoner, T.C.; Stygar, W.A.; Ives, H.C.; et al. Differential-output B-dot and D-dot monitors for current and voltage measurements on a 20-MA, 3-MV pulsed-power accelerator. Phys. Rev. Spec. Top.-Accel. Beams 2008, 11, 100401. doi:10.1103/PhysRevSTAB.11.100401.

21. Ma, G.; Li, C.; Quan, J.; Jiang, J.; Yin, Y.; Zhao, H.; Zhao, H. Measurement of VFTO on transformer entrance with transformer bushing sensor. Proc. CSEE 2010, 30, 122-128. doi:10.13334/j.0258-8013.pcsee.2010.33.002.

22. Metwally, I.A. D-dot probe for fast-front high-voltage measurement. IEEE Trans. Instrum. Meas. 2010, 59, 2211-2219. doi:10.1109/TIM.2009.2030928.

23. Qin, B.; Si, W.; Li, S.; Li, Y.; Liang, Y.; Qi, Z. Feasibility study of D-dot probe used for the detection of partial discharge. Electr. Meas. Instrum. 2016, 53, 108-112. doi:10.3969/j.issn.1001-1390.2016.06.020.

24. Bai, Y.; Wang, J.; Wei, G.; Yang, Y. Design and Simulation Test of an Open D-Dot Voltage Sensor. Sensors 2015, 15, 23640-23652. doi:10.3390/s150923640.

25. Zhao, P.; Wang, J.; Wang, Q.; Xiao, Q.; Zhang, R.; Ou, S.; Tao, Y. Simulation, Design, and Test of a Dual-Differential D-Dot Overvoltage Sensor Based on the Field-Circuit Coupling Method. Sensors 2019, 19, 3413. doi:10.3390/s19153413.

26. Kubo, T.; Furukawa, T.; Itoh, H.; Fukumoto, H.; Wakuya, H. Numerical electric field analysis of power status sensor observing power distribution system taking into account measurement circuit and apparatus. SICE Annual Conference 2011, 2011, pp. 2741-2746. doi:10.1541/ieejfms.131.171.

27. Sun, Z.; Ma, G.; Guo, P.; Chen, M.; Li, C.; Chen, W.; Ding, W.; Dai, M.; Wang, L. Design of a VFTO port hole sensor for $1000 \mathrm{kV}$ based on inner probe structure. High Volt. Eng. 2013, 39, 1426-1433. doi:10.3969/j.issn.1003-6520.2013.06.020. 
28. Wang, K.; Duan, Y.; Shi, L.; Qiu, S. Laboratory Calibration of D-dot Sensor Based on System Identification Method. Sensors 2019, 19, 3255. doi:10.3390/s19153255.

29. Wang, J.; Gao, C.; Yang, J. Design, Experiments and Simulation of Voltage Transformers on the Basis of a Differential Input D-dot Sensor. Sensors 2014, 14, 12771-12783. doi:10.3390/s140712771.

30. Gao, C.; Wang, J.; Yang, J.; Peng, H.; Ma, J. Experiment and simulation of D-dot voltage probe based on inverse problem of electric field. Trans. China Electrotech. Soc. 2016, 34, $36-42$. doi:10.19595/j.cnki.1000-6753.tces.2016.04.006.

31. He, W.; Luo, R.; Wang, J.; Ran, P.; Wang, Q.; Hou, X.; Zhou, K. Principles and experiments of voltage transformer based on self-integrating D-dot probe. Proc. CSEE 2014, 34, 2445-2451. doi:10.13334/j.0258-8013.pcsee.2014.15.015.

32. Luo, R. The Research and Design of Voltage Measurement Device Based on the Theory of Electric Field Coupling. Master's thesis, Chongqing University, Chongqing, China, 2014.

33. Xiong, Q.; Feng, X.; Gattozzi, A.L.; Liu, X.; Zheng, L.; Zhu, L.; Ji, S.; Hebner, R.E. Series Arc Fault Detection and Localization in DC Distribution System. IEEE Trans. Instrum. Meas. 2020, 69, 122-134. doi:10.1109/TIM.2019.2890892.

34. High Speed FET-Input Instrumentation Amplifier datasheet. Available online: https://www.ti.com/lit/ ds/symlink/ina111.pdf. (access on 27 September 2020). 